\title{
Criação de um bot para a conscientização de cuidados e impactos relacionados ao COVID-19
}

\author{
Washington P. Batista ${ }^{1,2}$, Kayo C. Santana ${ }^{1,2}$, Lenington C. Rios ${ }^{2}$, Claudia P. \\ Pereira $^{1,2}$ \\ ${ }^{1}$ Programa de Pós-Graduação em Ciência da Computação \\ ${ }^{2}$ Universidade Estadual de Feira de Santana (UEFS) - Feira de Santana, BA - Brasil \\ \{wstroks, kayosantana94, lenitorios\}@gmail.com, claudiap@uefs.br
}

\begin{abstract}
This paper presents COVID-19 bot, an alternative technology to spread information related to COVID-19 through Discord application. The developed bot aims to raise awareness of users by providing reliable information of the disease's growth. Here, we will present the developed features, which are already available to users and some information about how their data are collected. Hereafter, we aim to create new features and to enlarge information related to other diseases.
\end{abstract}

Resumo. Este artigo apresenta o bot COVID-19, uma tecnologia alternativa para a difusão de informações relacionadas ao COVID-19 através do aplicativo Discord. O bot desenvolvido almeja a conscientização de usuários com a disponibilização de informações verídicas relacionadas ao crescimento da doença. No trabalho, serão apresentados os comandos que já se encontram disponíveis e como as informações utilizadas são coletadas. Futuramente, almeja-se a ampliação desta ferramenta para novas funcionalidades $e$ informações também relacionadas a outras doenças.

\section{Introdução}

O COVID-19 foi detectado inicialmente em dezembro de 2019 como um surto local de pneumonia em Wuhan sem causa conhecida, com posterior determinação do novo coronavírus [Dong; Du and Gardner, 2020]. Desde então, o vírus se espalhou amplamente, não apenas por diversas províncias da China, como também para outros países, resultando na pandemia de COVID-19 [Dong; Du and Gardner, 2020].

Para controle da pandemia e amenização dos danos causados pela mesma, várias medidas sociais e de saúde pública, sejam elas individuais ou coletivas, estão sendo tomadas em diversos países. Algumas delas, salientadas pela WHO (World Health Organization), são: distanciamento físico entre pessoas, rastreamento de contágio, teste de casos suspeitos, isolamento e quarentena de pacientes infectados, restrições de aglomerações e promoção de medidas para incentivar, higienização constante das mãos e outras [WHO, 2020].

Dentre as medidas, a WHO (2020) destaca a importância do fornecimento de informações claras e acessíveis para a população, para que as medidas implementadas possam ser mais eficazes para o controle do surto, com o envolvimento total da sociedade. 
Neste contexto, surge a proposta de criação de um bot (também conhecido como robot ou web robot) para o aplicativo Discord (aplicativo de comunicação disponível em diferentes plataformas), denominado de COVID-19. Este bot foi desenvolvido com o objetivo de informar os usuários sobre diversas notícias atuais relacionadas à pandemia do COVID-19, extraídas de sites confiáveis, tais como: quantidade de casos totais, curados e óbitos em diferentes cenários (cidade, estado, global); informações sobre tratamento, diagnóstico e prevenção; contatos para solicitar atendimento e verificar a veracidade de informações, e, até mesmo, localização de um determinado tipo de médico em uma região (e.g. clínico geral, pneumologista e outros).

Nas seções seguintes, serão apresentadas algumas informações sobre a pandemia do COVID-19 e outros trabalhos semelhantes encontrados. Posteriormente, serão apresentados os comandos e o funcionamento do bot desenvolvido e, por fim, as considerações finais e os trabalhos futuros deste projeto.

\section{Impactos Relacionados ao COVID-19}

O coronavírus $(\mathrm{CoV})$ faz parte de uma grande família viral, que causa infecções respiratórias tanto em seres humanos quanto em animais, semelhantes a um resfriado comum [Xu et. al., 2020]. De acordo com WHO (2020), entretanto, alguns CoVs podem causar síndromes respiratórias graves, como a SARS - Severe Acute Respiratory Syndrome ou, no português, Síndrome Respiratória Aguda Grave, além do SARS-CoV (SARS associados ao coronavírus), e o coronavírus associado à Síndrome Respiratória do Oriente Médio (MERS - Middle East Respiratory Syndrome), conhecido como MERS-CoV [Xu et. al., 2020].

O novo coronavírus trata-se de um vírus ainda mais grave que o MERS-CoV e o SARS-CoV, por causa do seu rápido aumento de incidência e a crescente evidência de transmissão, tornando-o ainda mais contagioso [Grupo de Epidemiologia do Novo Mecanismo de Resposta de Emergência para Pneumonia por Coronavírus do Centro Chinês de Controle e Prevenção de Doenças, 2020, tradução nossa]. Denominado de COVID-19, ou SARS-CoV-2, o novo coronavírus possui forte afinidade com os receptores respiratórios humanos, o que sugere uma ameaça à saúde pública global [WHO, 2020].

De acordo com Xu et al. (2020), a disseminação do COVID-19 teve uma rápida expansão para a maior parte da China e para outros países em janeiro de 2020, totalizando, no mês de fevereiro de 2020, 37.589 pessoas infectadas no mundo todo. Além disso, o levantamento traz que, apesar do crescente número de casos, existe uma insuficiência nas investigações clínicas dos pacientes e poucos recursos para tratar os infectados [Xu et. al., 2020]. No mês de abril de 2020, o número de casos confirmados no mundo ultrapassa os 2,3 milhões, com um total de 158.422 mortos. No Brasil, o número de pessoas infectadas corresponde a 36.599 (cerca de 1,59\% do total de infectados no mundo) e 2.347 mortes [Johns Hopkins University, 2020].

\section{Trabalhos Correlatos}

Para esta seção, foram escolhidos dois trabalhos que possuem relação com o bot desenvolvido, ou seja, bots disponíveis no Discord que possuem a temática COVID-19, de modo que seja possível fazer um comparativo com o referido trabalho. Através do 
sistema de pesquisa disponibilizado pela plataforma, foi possível encontrar alguns que possuem tal semelhança. Tal plataforma ${ }^{1}$ é utilizada para a divulgação dessas ferramentas automatizadas no Discord, através da qual é possível pesquisá-los por tema ou título e convidar o bot desejado para um servidor.

Como critério de escolha desses dois trabalhos, analisou-se primeiro a quantidade de votos que cada bot possuía, referente ao parecer dos usuários, e em quantos servidores ativos ele estava incluso. Além disso, foi analisado o que cada um oferecia para o usuário de modo a ter semelhança com a proposta deste trabalho.

O primeiro trabalho, denominado de Coronavirus COVID- $19^{2}$, trata-se de um bot que envia atualizações com alertas de notificações e estatísticas de gráficos referentes ao novo coronavírus. Através dele, é possível visualizar todos os casos confirmados, listagens dos países afetados, informações sobre vários países ou regiões, além de ser possível ver as últimas notícias referentes ao COVID-19, que são atualizadas a cada uma hora. O gráfico exibido pelo bot mostra a curva de crescimento dos casos de pessoas infectadas, recuperadas, quantas estão ainda infectadas e quantidade de mortos.

O segundo bot, intitulado de Coronavirus Tracker $^{3}$, possui diferenças em relação ao primeiro citado. Uma delas é que, para exibir a curva em gráfico, é necessário requisitar por comando. Também é possível mostrar o histórico dos últimos seis dias do país informado, além dos dados estatísticos daquela localidade. Estes dois trabalhos correlatos, aqui apresentados, assim como os outros encontrados estão disponíveis apenas em inglês, tornando, desta maneira, o bot desenvolvido neste trabalho uma alternativa para a difusão de informações sobre o COVID-19 em português e com enfoque em dados que refletem a realidade brasileira.

\section{O Bot COVID-19}

Nesta seção, são apresentadas as tecnologias, os procedimentos, as funcionalidades e as fontes de informações empregados no desenvolvimento do bot COVID-19.

\subsection{Metodologia de desenvolvimento}

Para a elaboração e a construção do bot COVID-19, foi utilizada a tecnologia Node.js, que é um interpretador Javascript assíncrono de alta performance, projetado para criar aplicativos de rede escaláveis [OpenJS Foundation, 2020].

Como o intuito da proposta é a difusão da informação a respeito do vírus COVID-19, foram adotadas duas medidas para a extração dos dados. A primeira delas é o consumo de dados através de APIs (Application Programming Interface, no português Interface de programação de aplicações), disponibilizadas pelo próprio Governo Federal através da Lei de Acesso à Informação ou iniciativas colaborativas que oferecem repositórios de dados públicos em formato acessível, como por exemplo o brasil.io ${ }^{4}$. A

\footnotetext{
${ }^{1}$ Disponível através do endereço eletrônico: https://top.gg

${ }^{2}$ Informações encontradas em: https://top.gg/bot/682946560417333283

${ }^{3}$ Informações encontradas em: https://top.gg/bot/686329320440070211

${ }^{4}$ Disponível através do endereço eletrônico: https://brasil.io/home/
} 
segunda medida foi a criação de um procedimento de web scraping para dados em sites públicos.

Para a realização destes procedimentos, foram utilizadas as bibliotecas: Axios (para requisições no cliente http) e Cheerio (para selecionar a informação dos sites e imprimir o texto ou, até mesmo, o HTML selecionado da página).

Por fim, a execução do bot é realizada através do Discord que disponibiliza uma biblioteca chamada Discord.js para aplicações Node.js. O Discord é um aplicativo disponível para diferentes sistemas, que permite a comunicação de usuários através de voz, vídeo e texto. Foi desenvolvido, a princípio, para juntar pessoas através de diferentes jogos, mas vem sendo utilizado também para diversos outros objetivos, tais como clube escolar, grupo de jogadores, comunidade artística e comunicação entre amigos [Discord, 2020].

\subsection{Funcionamento do Bot}

Atualmente, o bot se encontra disponível no Discord e conta com 16 comandos para a obtenção de informações ou realização de ações relacionadas ao COVID-19. A Tabela 1 apresenta os comandos que podem ser utilizados para interações relacionadas ao COVID-19, suas descrições e seus identificadores (ID).

Tabela 1. Comandos do COVID-19

\begin{tabular}{|c|c|c|}
\hline ID & Comando & Descrição \\
\hline $\mathrm{C} 1$ & !ajuda & Exibe todos os comandos disponíveis \\
\hline $\mathrm{C} 2$ & !atendimento & Exibe número de telefone para atendimento sobre o COVID-19 \\
\hline $\mathrm{C} 3$ & !boletim & Boletim informativo do seu estado \\
\hline $\mathrm{C} 4$ & !brasil-covid19 & Exibe informações sobre quantidade de casos de COVID-19 no Brasil \\
\hline $\mathrm{C} 5$ & !cidade-covid19 & $\begin{array}{l}\text { Exibe informações sobre a quantidade de casos de COVID-19 em uma } \\
\text { cidade }\end{array}$ \\
\hline C6 & !diagnostico & Exibe informações sobre o diagnóstico do COVID-19 \\
\hline C7 & !estado-covid19 & $\begin{array}{l}\text { Exibe informações sobre a quantidade de casos de COVID-19 em um } \\
\text { estado }\end{array}$ \\
\hline $\mathrm{C} 8$ & !fakenews & $\begin{array}{l}\text { Exibe o número de telefone para verificar a veracidade de informações } \\
\text { relacionadas ao COVID-19 }\end{array}$ \\
\hline C9 & !fiqueemcasa & $\begin{array}{l}\text { Promove a interação de usuários para incentivar que os outros permaneçam } \\
\text { em casa. Exibe quantos usuários do servidor já participou da campanha. }\end{array}$ \\
\hline $\mathrm{C} 10$ & !global-covid19 & Exibe informações globais sobre casos relacionados ao COVID-19 \\
\hline $\mathrm{C} 11$ & !historia & Exibe informações sobre o surgimento do COVID-19 \\
\hline $\mathrm{C} 12$ & !medico & Exibe uma indicação de médicos em um determinado local \\
\hline $\mathrm{C} 13$ & !prevencao & Exibe informações sobre como se prevenir do COVID-19 \\
\hline C14 & !rendabasica & Exibe informações sobre o projeto de lei que institui o auxílio emergencial \\
\hline $\mathrm{C} 15$ & !transmissao & Exibe informações sobre formas de propagação do COVID-19 \\
\hline $\mathrm{C} 16$ & !tratamento & Exibe informações sobre o tratamento do COVID-19 \\
\hline
\end{tabular}


Para a utilização dos comandos, é necessário que um usuário inicialmente convide ${ }^{5} \mathrm{o}$ bot para o seu servidor. Todos os comandos podem ser executados através de um canal de texto do servidor, sendo que quase todos são executados de maneira semelhante à apresentada na Tabela 1, com exceção dos comandos C3, C5, C7 e C12 que precisam de parâmetros para realização de busca.

Os comandos C3 e C7 necessitam que o usuário digite o estado para a verificação de ocorrência de casos na região. Para tanto, o usuário deve utilizar o comando seguido da sigla do estado, para a realização da busca de seu interesse. Por exemplo, com o comando !estado-covid19 ba, o usuário solicita informações sobre ocorrências de casos na Bahia. Já para o C5, o usuário precisará informar a cidade sobre a qual deseja receber as informações. A busca para a cidade Salvador pode ser feita da seguinte maneira: !cidade-covid19 salvador.

Já para a busca de médicos (C12), é necessário também incluir a especialidade procurada. Assim, a busca é realizada da seguinte maneira: !medico p clínico geral I salvador-bahia. As letras "p" e "l" no comando indicam, respectivamente, o tipo de profissional e a localidade a serem procurados. No caso do exemplo citado, seriam procurados, na cidade de Salvador, médicos clínicos gerais.

\section{Considerações Finais}

O desenvolvimento e a publicação do bot COVID-19 para a plataforma Discord almejam a difusão de informações relacionadas à pandemia, auxiliando, através da seleção de informações de fontes confiáveis, a amenização dos seus efeitos e a propagação de conhecimentos importantes para seus usuários.

A escolha pelo Discord se deu em função da crescente quantidade de usuários desta plataforma. Segundo Hayashi (2018), em 2018 o Discord triplicou sua base de usuários em apenas um ano, atingindo a marca de 145 milhões de usuários, dos quais 19 milhões utilizavam o aplicativo diariamente. Vale ressaltar que os bots sobre o COVID19 encontrados nesta plataforma apresentam informações na língua inglesa, e que ter uma alternativa na língua portuguesa pode permitir a disseminação de informações sobre essa doença no território nacional, de maneira mais acessível.

Na situação vivenciada pelo surto do COVID-19, a conscientização e a adoção de medidas, tanto no âmbito pessoal quanto coletivo, são importantes como esforços para diminuir o contágio e os impactos causados pela doença, suavizando a possível superlotação de leitos, que pode acarretar o óbito de pacientes em casos mais graves devido à falta de atendimento.

Pretende-se, futuramente, expandir a proposta do bot COVID-19 para a disponibilização de novas informações relacionadas ao COVID-19 e também a implementação de novas funcionalidades, tais como: exibição de gráficos mostrando o crescimento de casos ao longo dos dias, comparação entre a forma de crescimento em diferentes locais e, até mesmo, estimativas para predição de crescimento de casos. Além

\footnotetext{
${ }^{5} \mathrm{O}$ convite pode ser realizado por qualquer administrador de um servidor através do endereço: https:/discordapp.com/oauth2/authorize?=\&client_id=699677964215910531\&scope=bot\&permissions= 8
} 
disso, almeja-se oferecer informações sobre outras doenças, como, por exemplo, dengue, chikungunya e zika, e que este bot seja acessado através de outras plataformas digitais, tornando-o ainda mais acessível para a população.

\section{Referências}

Discord (2020). Discord - Chat for communities and friends. Disponível em: $<$ https://discordapp.com/>. Acesso em: 14 Abril. 2020.

Dong, E.; Du, H.; Gardner, L. (2020). An interactive web-based dashboard to track COVID-19 in real time. The Lancet infectious diseases. Disponível em: $<$ https://www.thelancet.com/journals/laninf/article/PIIS1473-3099(20)301201/fulltext>. Acesso em: 21 Abr. 2020.

Hayashi, E. (2018). Discord quase triplica a sua base de usuários em apenas um ano. Disponível em: <https://canaltech.com.br/apps/discord-quase-triplica-a-sua-base-deusuarios-em-apenas-um-ano-113878/>. Acesso em: 15 Abr. 2020.

Johns Hopkins University. (2020). COVID-19 Dashboard by the Center for Systems Science and Engineering (CSSE) at Johns Hopkins University (JHU). Disponível em: $<$ https://coronavirus.jhu.edu/map.html $>$. Acesso em: 24 Abr. 2020.

OpenJS Foundation (2020). About Node.js. Blog Optus Software. Disponível em: $<$ https://nodejs.org/en/about/> Acesso em: 03 Abr. 2020.

Grupo de Epidemiologia do Novo Mecanismo de Resposta de Emergência para Pneumonia por Coronavírus do Centro Chinês de Controle e Prevenção de Doenças. (2020). Características epidemiológicas da nova pneumonia por coronavírus. Chinese Journal of Epidemiology. Disponível em: $<$ http://rs.yiigle.com/yufabiao/1181998.htm>. Acesso em: 10 Abr. 2020.

Who - World Health Organization (2020). Coronavirus disease 2019 (COVID-19): situation report, 72 .

Xu, X.; et. al. (2020). Clinical findings in a group of patients infected with the 2019 novel coronavirus (SARS-Cov-2) outside of Wuhan, China: retrospective case series. bmj, v. 368. 